\title{
Psychosocial Aspects of Familial Breast and Ovarian Cancer: Psychological Guidelines for Genetic Testing
}

\author{
M. Decruyenaere ${ }^{1, \#}$, \\ G. Evers-Kiebooms ${ }^{1}$, E. Claes ${ }^{1}$, \\ L. Denayer ${ }^{1}$, M. Welkenhuysen ${ }^{1}$, \\ E. Legius ${ }^{1}$ and K. Demyttenaere ${ }^{2}$ \\ ${ }^{1}$ Center Human Genetics, University Leuven, \\ Leuven, Belgium \\ ${ }^{2}$ Department of Psychiatry, University \\ Leuven, Leuven, Belgium
}

\begin{abstract}
Genetic conditions such as hereditary breast/ovarian cancer (HBOC) evoke strong emotions such as anxiety, depression, anger and guilt for passing the defect to the offspring. Research showed that first degree relatives of breast cancer patients reported impairment in daily functioning and sleep disturbances due to breast cancer worry. The perception of personal genetic susceptibility to HBOC is particularly burdensome. People react with different coping strategies to handle these emotions, such as minimisation of the problem, denial or hypervigilance (emotion-focussed coping). People not only react emotionally, but also cognitively to the threatening genetic condition. They construct a cognitive representation of the health threat (e.g., causes, symptoms and severity of the disease, susceptibility to the disease), based on their current and past experiences with the disease. They also generate possible action plans for managing the threat, such as seeking medical
\end{abstract}

\footnotetext{
\# Corresponding author: M. Decruyenaere, PhD, Center Human Genetics, Herestraat 49, 3000, Leuven, Belgium, Email: marleen.decruyenaere@ med.kuleuven.ac.be
}

information or deciding to have a predictive test (problem-focussed coping). The interaction between the rational-cognitive and the subjectiveemotional processes is very complex. The literature on HBOC has reported inconsistent findings about the relation between emotional aspects of threat (such as anxiety) and health related actions (such as screening behaviour). In some studies, cancer anxiety was associated with less engagement in screening, while other research found that anxiety was unrelated to health screening utilisation. Some studies also reported that cancer-specific distress was a stimulating factor to engage in health behaviour. The conflict-theory states that extremely high and extremely low levels of distress lead to defective coping patterns, while moderate levels are more likely to produce more effective responses.

Genetic conditions evoke strong emotions because they are perceived as ego threatening and stigmatising. The familial disease and the genetic risk can undermine one's self-esteem and identity. This is potentially more serious for daughters of breast-cancer patients, who were in adolescence or childhood when their mother was diagnosed. At that moment, they are undergoing a phase of ego and sexual development, in which the identification with the mother and the female body is of utmost importance. The breast/ovarian cancer in the mother (and in other female members of the family), combined with the own genetic susceptibility, becomes a threat to the daughter's body image, emotional growth, selfesteem and sexual identity.

Genetic conditions affect a whole family, not just one individual. Family members develop 
different ways of coping with the familial disease and the genetic susceptibility. Some cope by seeking medical information and sharing their experiences and feelings with relatives or important others, while others deny the seriousness of the women's disease and refuse to discuss any aspect of the situation with other family members. Some families even develop family secrets and magical thinking about the family disease and the family risk (such as patient preselection). Communication in the family is important, not only for the dissemination of medical/genetic information, but also for building a social support network. The communication may however be hindered by emotional coping mechanisms of family members. The "messenger in the family", the "first applicant" for a DNA-test or for a breast amputation and the "obligate carrier" are particularly vulnerable. Another difficulty is that HBOC is generally perceived as mere women's problem. This, in combination with the taboo surrounding cancer, sexuality and fertility, can result in a neglect of the male family members in the family communication process. Intergenerational conflicts or family rifts may further complicate the communication in the family.

Important protective factors against overwhelming feelings are the individual's personal strength (self-confidence, self-efficacy, feelings of control) and the individual's social network (in childhood and in later life).

The approach to predictive testing for HBOC in Leuven is multidisciplinary and is based on the above-mentioned psychological concepts [1]. Given the complexity and uncertainties of HBOC and given the potential impact of HBOC on emotions, self-esteem and family functioning, we offer the applicants a combination of information-oriented and psychological counselling. Both are prerequisites for free informed decisionmaking. The medical/educational counselling, combined with emotional support counselling, is the essential part of the first counselling session. The test participants also receive an educational text about HBOC and its inheritance, about cancer risks, the predictive test and recommendations concerning prevention and early detection. After this session, a medical examination of the breasts and the ovaries is requested. The major issue of the second counselling session is psychological counselling which consists of three (interacting) dimensions: (a) individual emotional support, (b) decision counselling and (c) support of the family communication. Decision counselling includes a discussion with the test participants about their cognitive and emotional appraisal of the situation, their coping strategies and their psychological and social abilities and resources. After this session, psychometric questionnaires are administered. The main aim of this psychological assessment is to establish a baseline evaluation of each individual, which is important in the context of a longitudinal study to investigate psychological research questions, such as the impact of anxiety on adherence to surveillance programmes. All data concerning the participant, including the results from the psychological assessment, are discussed by the predictive testing team to identify possible problems or pitfalls. In the third session the relevant aspects are discussed with the test participant and a blood sample is taken. The counselling of male family members requesting a predictive test, is reduced to 2 sessions (planned on one day) because the medical risks are smaller. After disclosure of the predictive test result, short- and long-term medical counselling and psychosocial support are provided. If a woman is considering preventive mastectomy, additional counselling is provided before and after the surgery.

\section{References}

[1] Decruyenaere, M., Evers-Kiebooms, G., Denayer, L., Welkenhuysen, M., Claes, E., Legius, E. and Demyttenaere, K. Predictive testing for hereditary breast and ovarian cancer. A psychological framework for pre-test counselling. European Journal Human Genetics (in press). 


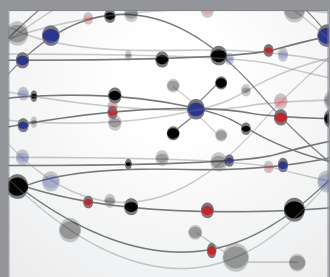

The Scientific World Journal
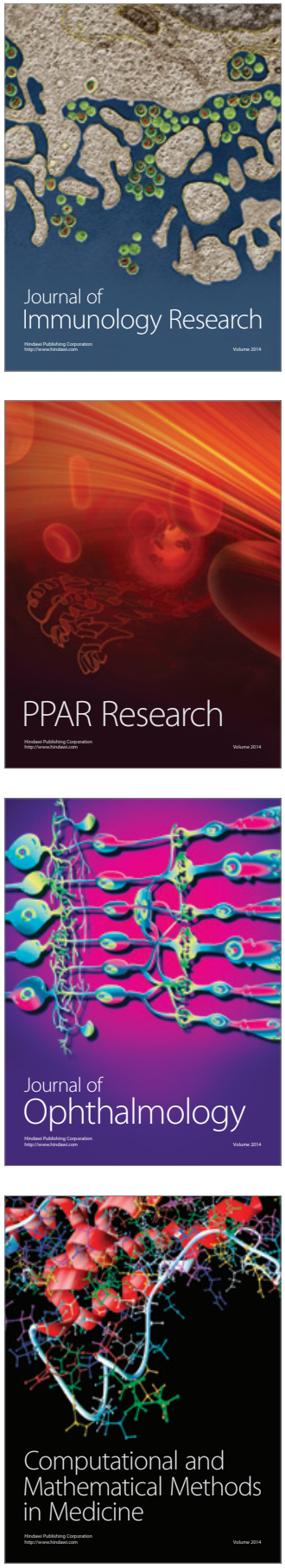

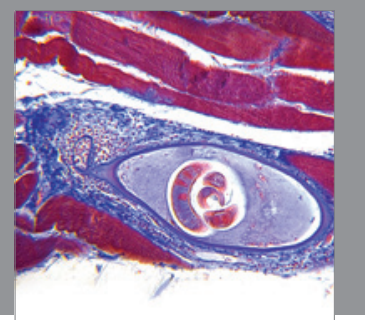

Gastroenterology

Research and Practice
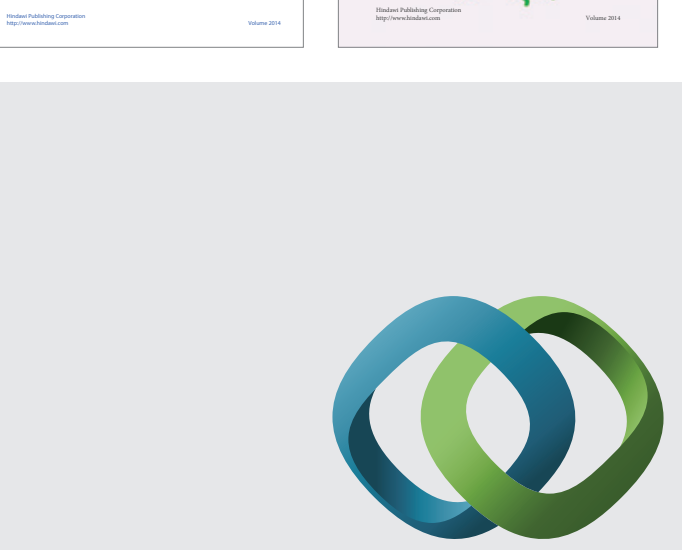

\section{Hindawi}

Submit your manuscripts at

http://www.hindawi.com
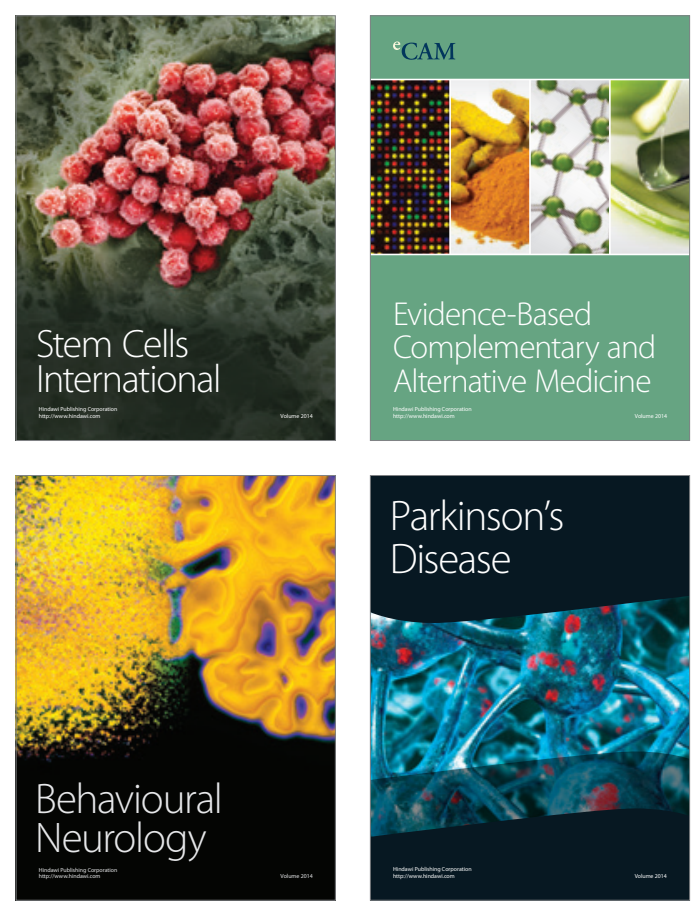

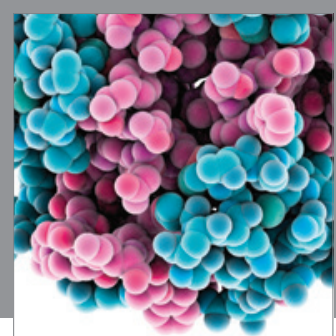

Journal of
Diabetes Research

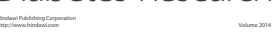

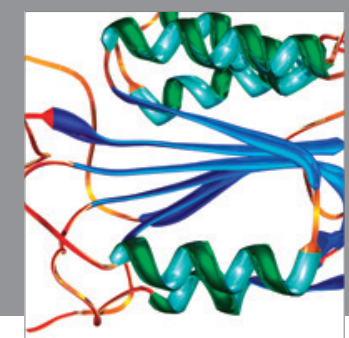

Disease Markers
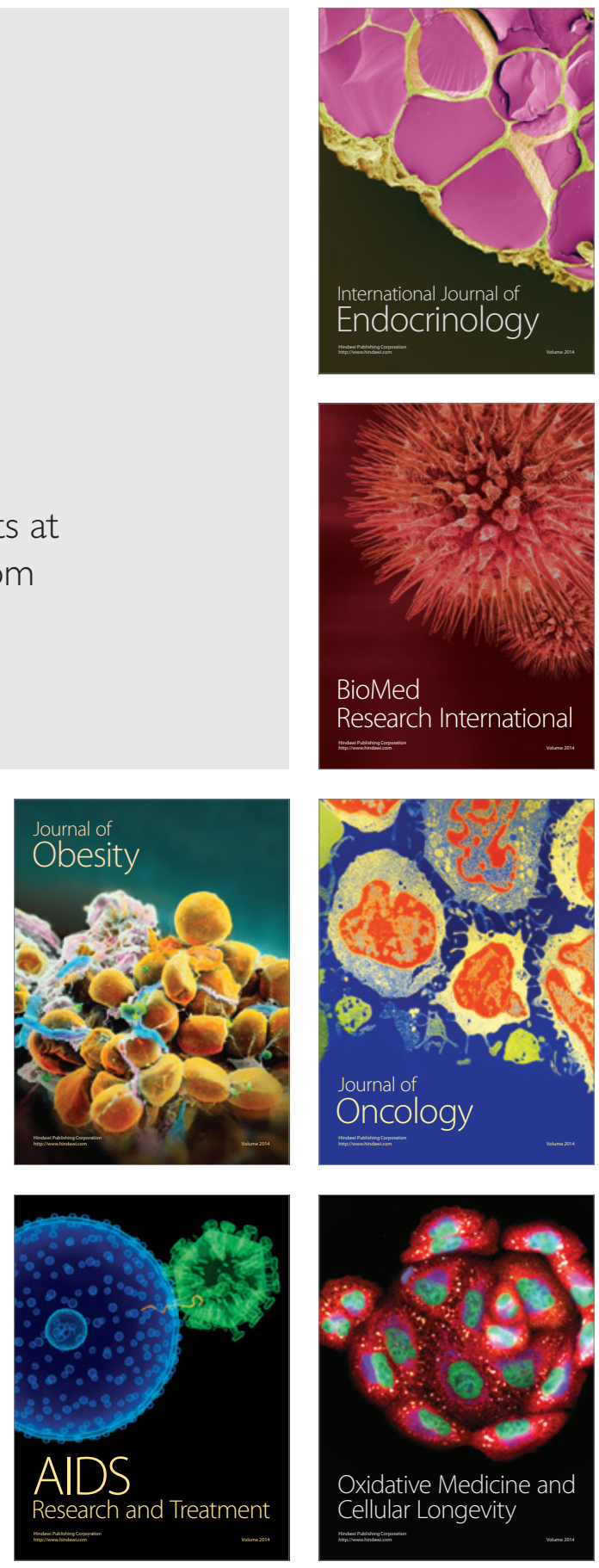